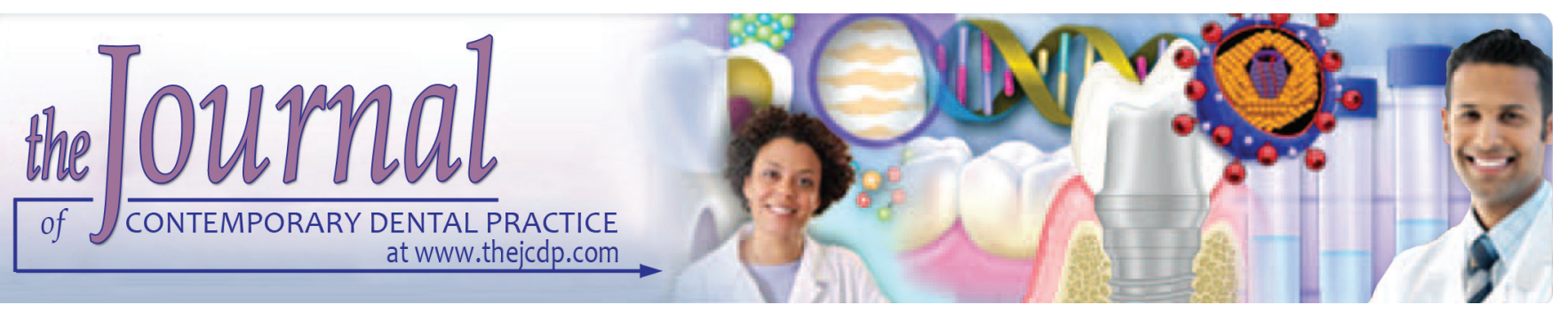

\title{
Influence of Light-curing Units on Surface Microhardness and Color Change of Composite Resins after Challenge
}

\author{
${ }^{1}$ Morganna B de A Souza, ${ }^{2}$ André LF Briso, ${ }^{3}$ Bruna de Oliveira-Reis, ${ }^{4}$ Paulo H Dos Santos, ${ }^{5}$ Ticiane C Fagundes
}

\begin{abstract}
Aim: The aim of this study was to evaluate microhardness and color change $(\Delta E)$ of composite resins, light cured with different light emission diodes (LEDs) and submitted to artificial accelerated aging (AAA).
\end{abstract}

Materials and methods: Two composite resins with lucirinTPO photoinitiator were selected: Tetric N-Ceram (Ivoclar Vivadent, A1) and Vit-I-escence (Ultradent, WO).A resin with the only camphorquinone photoinitiator was chosen as a negative control: Filtek Z350XT (3M ESPE, WD). Disc-shaped specimens were prepared ( $5 \mathrm{~mm}$ diameter; $1.5 \mathrm{~mm}$ thick) and photopolymerized with an LED with one wavelength (Radii-Cal, $\mathrm{SDI}$ ) or multiple wavelengths (Valo, Ultradent), for each composite resin $(n=10)$. Surface microhardness and color evaluations were performed immediately after specimen preparation and after AAA. Microhardness results were analyzed using Kruskal-Wallis and Mann-Whitney tests for multiple comparisons. To compare the evaluation of microhardness at different times, the Wilcoxon test was used. Mean values of $\Delta \mathrm{E}, \Delta \mathrm{L}, \Delta \mathrm{a}$, and $\triangle b$ were evaluated using two-way analysis of variance (ANOVA), and Tukey test for multiple comparisons $(\alpha=0.05)$.

Results: Regarding microhardness, a statistically significant difference between the two LEDs was observed for Vit-Iescence after AAA. When comparing composite resins that were light-cured with the same device, FiltekZ350XT obtained the greatest microhardness. All groups presented a statistically significant decrease in microhardness from the initial time to the AAA. Regarding $\triangle E$, no statistically significant difference between the two LEDs was observed. When comparing composite resins, FiltekZ350XT showed the highest $\Delta \mathrm{E}$ values.

${ }^{1-3,5}$ Department of Restorative Dentistry, UNESP - São Paulo State University, Araçatuba School of Dentistry, Araçatuba, SP, Federative Republic of Brazil

${ }^{4}$ Department of Dental Materials and Prosthodontics,UNESP São Paulo State University, Araçatuba School of Dentistry,, Araçatuba, SP, Brazil

Corresponding Author: Ticiane C Fagundes, Department of Restorative Dentistry, UNESP - São Paulo State University, Araçatuba School of Dentistry, Araçatuba, SP, Federative Republic of Brazil, Phone: +55 18 3636-3253, e-mail: ticiane@ foa.unesp.br
Conclusion: In general, an LED with multiple wavelengths influenced the microhardness of only one resin containing lucirin-TPO after AAA. The $\triangle E$ was more influenced by the composite resin than the LED device.

Clinical significance: The knowledge of composite resin with deficiencies in the polymerization mechanism could contribute to preventing restorations to become more susceptible to color change and reduction of the mechanical strength.

Keywords: Composite resin, Curing lights, Dental, Photoinitiators.

How to cite this article: Souza MBdeA, Briso ALF, Oliveira-Reis $\mathrm{B}$, Dos Santos PH, Fagundes TC. Influence of Light-curing Units on Surface Microhardness and Color Change of Composite Resins after Challenge. J Contemp Dent Pract 2019;20(2):204-210.

Source of support: São Paulo Research Foundation (FAPESP), grant number 2014/24478-0.

Conflict of interest: None

\section{INTRODUCTION}

Camphorquinone has been traditionally used as a photoinitiator in composite resins and has an activation peak at a wavelength around $460 \mathrm{~nm}$. However, its intense yellow color has limited its use, particularly in resin materials used for bleached teeth. ${ }^{1}$ Thus, other photoinitiators have been used for these materials, such as phenylpropanodione (PPD) and lucirin-TPO. ${ }^{1-3}$

A compositeresin with deficiencies in the polymerization of the organic matrix undergoes degradation and thus changes in its mechanical properties, becoming more susceptible to color change and a reduction in mechanical strength. ${ }^{4}$

Thus, researchers have submitted composite resins to challenges such as AAA, to simulate degradation that occurs in a composite resin restoration in the oral cavity. ${ }^{5}$ The AAA is a type of radiation that has a photo-oxidation potential that induces the breakdown of single or carbon double bonds. ${ }^{5}$ These chemical bonds have an important function in the configuration of the polymer chains present in the organic matrix of the resin base material. ${ }^{5}$ 
Recently, a new generation of LED was launched with multiple wavelengths. ${ }^{2,6}$ This new technology, in contrast to the previous versions, emits a light spectrum which varies in wavelength from $385-515 \mathrm{~nm}^{2,3}$ This feature permits the curing of resin materials even when different photoinitiators are used in their compositions. ${ }^{2,7,8}$ The literature is scarce in studies evaluating the microhardness and color change of commercial composite resins when using LEDs with multiple wavelengths.

The aim of this study was to evaluate the microhardness and color stability of commercial composite resins with different photoinitiators, light cured using two types of LEDs, before and after AAA. The first null hypothesis tested was that there was no statistical difference in surface microhardness and color change between two generations of light curing units (LCUs) when they were used for curing commercial composite resins with different photoinitiators after the proposed challenge. Furthermore, the second null hypothesis tested was that there was no statistical difference in surface microhardness and color change among different tested composite resins when photocured with single-wave or poly-wave LCUs, before and after a proposed challenge. The third null hypothesis was that there was no statistical difference in surface microhardness when comparing results before and after AAA for each condition studied.

\section{MATERIALS AND METHODS}

\section{Specimen Preparation}

To evaluate the effect of light curing using two types of LEDs on surface microhardness, three composite resins were used. The inclusion criteria of resins were the presence of luricin-TPO, associated or not with camphorquinone, and the absence of this compound as a photoinitiator was excluded. Two composite resins contained camphorquinone and lucirin-TPO in their compositions: Tetric-N-Ceram shade A1 (Ivoclar Vivadent, Schaan, Liechtenstein) and Vit-l-escence shade WO (Ultradent Products Inc., South Jordan, UT, USA). ${ }^{7}$ The remaining composite resin, which contained only camphorquinone (information confirmed by manufacturer),was used as a negative control: Filtek Z350XT shade WD (3M ESPE Dental Products ${ }^{\mathrm{TM}}$, St. Paul, MN, USA). Additionally, two types of LED devices were used: single wavelength (Radii-Cal, SDI, Victoria, Australia) and multiple wavelengths (Valo, Ultradent, Products Inc., South Jordan, UT, USA). Composite resins and LED devices are described in Table 1.

Ten discs for each material were obtained using a Teflon matrix ( $5 \mathrm{~mm}$ in diameter $\times 1.5 \mathrm{~mm}$ thick). Resin composite was inserted into the matrix in one increment that was covered with a glass slide to allow excess resin runoff and protect against the formation of an oxygen inhibition layer. Photocuring was performed for 40 seconds using the LED device of the study group. The wavelength apparatus of each LED was measured using a spectroradiometer (Model 77702-Oriel Instruments, Danbury, CT, USA), and power with the use of a radiometer (Radiometer LED-Demetron/ Kerr, Danbury, CT, USA) (Table 1).

Finishing and polishing were performed using silicon carbide sandpaper in decreasing granulation ( $\# 600$, \#800 and \#1200). Diamond polishing paste $(1 \mu \mathrm{m})$ was applied using a felt disc for 60 seconds (APL-4, Arotec S.A. Ind Com, Cotia, SP, Brazil). The samples were washed for 10 minutes in an ultrasonic tub (Cristófoli, Campo Mourão, SP, Brazil) to remove residues found on the body surface of the specimen after each change of sandpaper or disk

Table 1: Composite resins and curing light LEDs used in this study

\begin{tabular}{|c|c|c|c|c|c|}
\hline Materials & Color & Composition & Mean particle size & $\%$ Filler (by vol) & Manufacturer \\
\hline $\begin{array}{l}\text { Tetric-N-Ceram } \\
\text { (nanohybrid) }\end{array}$ & $\mathrm{A} 1$ & $\begin{array}{l}\text { Bis-GMA, UDMA, TEGDMA, } \\
\text { barium glass, barium glass and } \\
\text { aluminum, highly disperse silica, } \\
\text { mixed oxides, prepolymers }\end{array}$ & $40 \mathrm{~nm} 3000 \mathrm{~nm}$ & $55-57$ & $\begin{array}{l}\text { IvoclarVivadent, } \\
\text { Schaan, } \\
\text { Liechtenstein }\end{array}$ \\
\hline $\begin{array}{l}\text { Vit-l-escence } \\
\text { (microhybrid) }\end{array}$ & WO & $\begin{array}{l}\text { Bis-GMA, barium } \\
\text { borosilicate and other fillers, } \\
\text { camphorquinone, an amine co- } \\
\text { initiator and a proprietary initiator }\end{array}$ & $0.7 \mu \mathrm{m}$ & 58 & $\begin{array}{l}\text { UltradentProducts } \\
\text { Inc., South Jordan, } \\
\text { UT, USA }\end{array}$ \\
\hline $\begin{array}{l}\text { FiltekZ350XT } \\
\text { (nanofill) }\end{array}$ & WD & $\begin{array}{l}\text { Bis-GMA, Bis-EMA, UDMA, } \\
\text { TEGDMA, zirconia, silica and } \\
\text { camphorquinone }\end{array}$ & $\begin{array}{l}5-20 \mathrm{~nm} \mathrm{n} \text {. ag. } \\
4-11 \mathrm{~nm} \text { ag. }\end{array}$ & 59.5 & $\begin{array}{l}\text { 3M/ESPE Dental } \\
\text { Products, St Paul, } \\
\text { MN, SA }\end{array}$ \\
\hline Curing Light & Type & Wavelength & Intensity & & Manufacturer \\
\hline Radii-Cal & Singlewave & $440-480 \mathrm{~nm}$ & $\sim 1200 \mathrm{~mW} / \mathrm{cm}^{2}$ & & $\begin{array}{l}\text { SDI Limited, } \\
\text { Victoria, Australia }\end{array}$ \\
\hline Valo & Polywave & $395-480 \mathrm{~nm}$ & & & $\begin{array}{l}\text { UltradentProducts } \\
\text { Inc., South Jordan, } \\
\text { UT, USA }\end{array}$ \\
\hline
\end{tabular}

Bis-GMA, bisphenolA-glycidyl methacrylate; UDMA, urethane dimethacrylate; TEGDMA, triethylene glycol dimethacrylate; Bis-EMA, bisphenol A ethoxylate dimethacrylates 
felt. The specimens were kept at 100\% relative humidity and $37^{\circ} \mathrm{C}$ for 24 hours.

\section{Surface Microhardness Analysis}

Samples were subjected to microhardness analysis (Shimadzu HMV 2000, Shimadzu Corporation, Kyoto, Japan) using a Knoop diamond under a $25 \mathrm{~g}$ for $30 \mathrm{~s}$. Five indentations in the central region of the sample surface were made with a $100 \mu \mathrm{m}$ distance between each indentation. The arithmetic mean was then calculated.

\section{Color Change}

Color Change $\Delta \mathrm{E}$ analysis was performed using a reflection spectrophotometer (UV Visible, Model UV-2450, Shimadzu, Kyoto, Japan). The $\Delta \mathrm{E}$ was calculated using the CIE $\mathrm{L}^{*} \mathrm{a} * \mathrm{~b} *$, established by the ComissionInternacionaleI'Eclairaga-CIE. The CIE L*a ${ }^{*} \mathrm{~b}$ * allowed for the specification of color perceptions in terms of three-dimensional space by reflection vs. wavelength. The " $\mathrm{L}$ " axis is known as luminosity and extends from 0 (black) to 100 (perfect white). The coordinate "a" represents the amount of red (positive values) and green (negative values), while the coordinate " $b$ " is the amount of yellow (positive value) and blue (negative values). The coordinates " $a$ " and " $b$ " coexist on the same plane in this three-dimensional space. Five readings were taken for each surface; an average of the readings was obtained from the area that was evaluated. The samples were always positioned in the same way to standardize the reading area and avoid possible variations.

\section{Challenge}

The specimens from each experimental group were submitted to the AAA process performed in an aging chamber using UVB /condensation (Equilam, Diadema, SP, Brazil) according to ASTM G 53 (American Society for Testing Materials, Standard 53). This process consisted of ultraviolet light in alternating periods ( 8 hours) and moisture (4 hours), under heat $\left(65 \pm 3^{\circ} \mathrm{C}\right.$ or $\left.45 \pm 3^{\circ} \mathrm{C}\right)$ and $100 \%$ humidity. The samples were subjected to a total of 252 hours of aging and 168 hours of UVB radiation with a peak emission of $313 \mathrm{~nm}$.

\section{Final Surface Microhardness and Color Analysis}

The same methodology for surface microhardness and color analysis were performed as described above. The differences in the values of $L^{*}(\Delta L), a^{*}(\Delta a)$, and $b^{*}(\Delta b)$ were determined, and the overall change in color $(\Delta \mathrm{E})$ was also calculated. The CIE $\mathrm{L}^{*} \mathrm{a}{ }^{*} \mathrm{~b} *, \Delta \mathrm{E}$ was calculated using the formula: $\Delta \mathrm{E}=[(\Delta \mathrm{L}) 2+(\Delta \mathrm{a}) 2+(\Delta \mathrm{b}) 2]^{1 / 2}$.

\section{Statistical Analysis}

The software StatView version 5.0 (SAS Institute, Cary, NC, USA) was used for the statistical analysis. The power analysis was performed to determine the sample size for each experiment group, which determined that $\mathrm{n}=10$ provided a potency of at least 0.8 at a significance level of 0.05 .

The assumptions of equality of variances and normal distribution of data were checked using the Bartlett and Shapiro-Wilk tests, respectively. Since homogeneity was not achieved, the microhardness results were analyzed using the Kruskal Wallis test, followed by the Mann Whitney test for multiple comparisons. The Wilcoxon test was used to compare the microhardness at the evaluation times. The mean values of $\Delta \mathrm{E}, \Delta \mathrm{L}, \Delta \mathrm{a}$, and $\Delta \mathrm{b}$ were evaluated using two-way analysis of variance (ANOVA), while the Tukey test was used for multiple comparisons. The level of significance was set at $5 \%$.

\section{RESULTS}

No statistically significant difference was found between the two LEDs for the microhardness results, except that Vit-l-escence presented greater values of microhardness when photopolymerized with the poly-wave device after AAA (Table 2). The microhardness results before AAA showed that Tetric-N-Ceram exhibited a lower surface microhardness when compared to other materials, independent of the photo-activating apparatus used (Table 2). After AAA, FiltekZ350XT maintained superior values when compared to the other materials and using the single-wave device; however, Filtek Z350XT was similar to Vit-l-escence when using the poly-wave device (Table 2). The Wilcoxon test showed that there was a statistically significant difference between the before and after AAA for all groups.

Regarding all color parameters used, no resin composite suffered from the influence of the type of LCU

Table 2: Results regarding the baseline and final microhardness measurement of groups submitted to accelerated artificial aging

\begin{tabular}{lllll}
\hline & & Tetric N-Ceram & Vit-l-escence & Filtek Z350XT \\
\hline Baseline & Radii-Cal & $58.08 \pm 3.81 \mathrm{bB}$ & $72.40 \pm 3.63 \mathrm{bA}$ & $83.80 \pm 5.60 \mathrm{bA}$ \\
& Valo & $61.23 \pm 5.54 \mathrm{bB}$ & $75.74 \pm 5.78 \mathrm{bA}$ & $82.68 \pm 6.66 \mathrm{bA}$ \\
Final $^{*}$ & Radii-Cal & $47.59 \pm 2.34 \mathrm{bB}$ & $49.10 \pm 2.63 \mathrm{bB}$ & $60.45 \pm 4.64 \mathrm{bA}$ \\
& Valo & $49.43 \pm 2.03 \mathrm{bB}$ & $58.15 \pm 1.63 \mathrm{aA}$ & $58.07 \pm 5.37 \mathrm{bA}$ \\
\hline
\end{tabular}

Equal letters indicate no statistically significant differences. Lowercase referring in columns when the same period was considered. Uppercase referring in rows. ${ }^{*}$ Indicates a statistically significant decrease between the baseline and final periods 
Influence of Light-curing Units on Surface Microhardness and Color Change of Composite Resins after Challenge

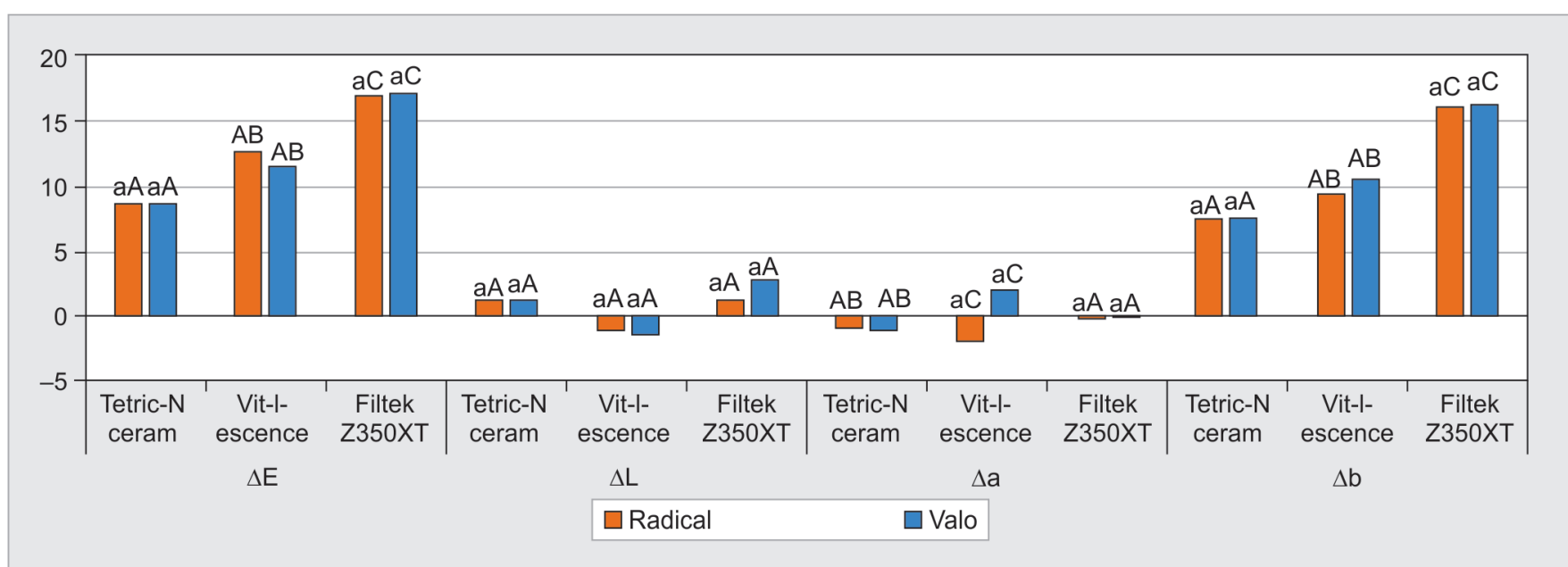

Graph 1: The overall color change of groups submitted to artificial accelerated aging, for each of the dental composite resins. Equal letters indicate no statistically significant differences. Lowercase referring between LCUs, and uppercase among resins when the same LCU eas used

after AAA (Graph 1). When comparing the composite resins, statistical superiority of the $\Delta \mathrm{E}$ values was observed in the following sequence: FiltekZ350>Vit-1escence $>$ TetricN-Ceram, regardless of the LCU used after AAA (Graph 1). The $\Delta \mathrm{L}$ values presented no statistically significant differences for allcomposite resins for both LCUs (Graph 1). Statistical superiority of the $\Delta \mathrm{a}$ values in the following sequence was observed for both LCUs: Vit-l-escence > Tetric N-Ceram > FiltekZ350XT (Graph 1). Statistical superiority of the $\Delta b$ values was observed in the following sequence for both LCUs: FiltekZ350XT > Vit-l-escence > Tetric N-Ceram (Graph 1).

\section{DISCUSSION}

The evaluation of some mechanical and physical properties can provide an indirect measure to preview the degree of conversion of the monomers present in composite resins, to include micro-hardness and overall color change. ${ }^{9}$ Surface hardness is related to material wear resistance and the ability to maintain form stability. Two tests are usually used for superficial microhardness measurements, being: Vickers and Knoop. The Knoop method is more used for the measurement on very small areas, and more fragile materials so were the choice for the present study. About the color evaluation, the best methods for recording color changes are the CIE L* $\mathrm{a}^{*}$ $b^{*}$ and the CIE L $a^{*} b * 2000$. In this study, the first one was chosen because of the larger number of references in the literature to compare results.

The first null hypothesis tested was rejected, since the microhardness results after AAA showed that Vitl-escence presented statistically superior values when the poly-wave LCU was used. It is believed that these results were found because of Vit-l-escence contains camphorquinone and lucirin-TPO; making thepolywave
LCU more effective. ${ }^{4}$ These results were similar to those observed by Santini et al., ${ }^{7}$ where the same resin (Vit-l-escence) showed better microhardness values and degree of conversion when a poly-wave LED LCU was used. Schneider et al. ${ }^{10}$ showed that formulations containing TPO exhibited higher reactivity than those with camphorquinone when polymerized with a quartztungsten-halogen LCU. Furthermore, the photochemical process for TPO leads to the cleavage of the C-P bond, forming acyl and phosphonyl radicals; the compounds derived from acyl phosphine oxides have higher molar absorptivity than camphorquinone, which means that those compounds absorb more light than the diketone molecule (camphorquinone) at the corresponding maximum peak absorption. ${ }^{2}$ However, Tetric-N-Ceram resin also has the same photoinitiators presented in Vit-l-escence. ${ }^{11}$ This difference of results from both resins may be due to the different amount of the initiators in the formulations; unfortunately, the manufacturers do not disclose this information. However, a study conducted by Sim et al. ${ }^{11}$ showed that there was no difference in the comparison of the two types of LEDs since the devices with multiple wavelengths reached similar values of microhardness, polymerization shrinkage, flexural and compressive properties as single-wave devices. ${ }^{11}$ Similar results occurred for all conditions studied, except for Vit-l-escence after AAA as cited above.

With regards to the composite resins when photopolymerized by the same LCU, superior microhardness was found for the nanoparticulate resin, FiltekZ350XT, causing the second null hypothesis to be rejected. There are two presentation forms of the inorganic particles in all nanoparticulate resins: dispersed-silica particles, measuring about $20 \mathrm{~nm}$; and adhering, silica-zirconia nanocomplexes, which behave as a single structure, 
measuring an average of $75 \mathrm{~nm}$. The union of these two particles provides a greater resistance for the material, which is different from nanohybrid and microhybrid resins, which tend to be less resistant because they have fewer filling agents. ${ }^{12}$ A previous study ${ }^{13}$ showed that the size of the filler, the weight of the filler content, and the shape and contact surface between nanofillers and the organic phase has a direct relation with the mechanical properties of nanoparticulate composite resins. Santini et al. ${ }^{7}$ also found that Vit-l-escence had greater surface microhardness when compared with Tetric-N-Ceram, as observed in baseline results from the present study, despite Teric N-Ceram is a nanohybrid and Vit-l-escence is a micro-hybrid composite resin.

There was a statistically significant decrease in microhardness values between both periods studied, for all groups submitted to AAA, rejecting the third null hypothesis. These results corroborate with Catelan et al., ${ }^{14}$ who analyzed the Vit-l-escence composite resin and observed a reduction in surface microhardness after AAA. However, in a study performed by Reis et al. ${ }^{15}$ demonstrated that AAA did not change the mechanical and physical properties, such as hardness, compressive strength, thermal behavior, and spectral profile, of Tetric $\mathrm{N}$-Ceram. It is important highlighted that study used a $50 \mathrm{kgf}$ load for 5 seconds, which was different from the study conducted by Catelan et al. ${ }^{14}$ and the present study which used equivalent parameters, $50 \mathrm{kgf}$ load for 15 seconds and a $25 \mathrm{kgf}$ load for 30 seconds, respectively. ${ }^{14,15}$

Various methods can be used to assess colors, such as the use of guide scales, image analysis, colorimeters, or spectrophotometers. Since the use of spectrophotometers allows for objective assessment and provides accurate quantitative data, it was the method utilized in this study. $^{16}$

The parameter, $\Delta \mathrm{E}$, indicates the relative color changes that an investigator can use for the materials before and after treatment or between intervals. According to Inokoshi et al. ${ }^{17}$, values of $\Delta \mathrm{E}<1$ are regarded as not appreciable by the human eye. Values $1<\Delta \mathrm{E}<3.3$ are considered appreciable by skilled operators, but clinically acceptable, whereas values of $\Delta \mathrm{E}>3.3$ are considered appreciable by non-skilled persons, and are not clinically acceptable. Therefore, color changes above a value of $\Delta \mathrm{E}$ $=3.3$ were considered clinically unacceptable. Although the tests were conducted under stringent conditions, the average $\Delta \mathrm{E}$ of all the samples submitted to AAA was greater than what is considered clinically acceptable (i.e., $\Delta \mathrm{E}>3.3){ }^{17}$

There was no statistically significant difference for all $\Delta$ analyzed in the present study with regards to the LUCs used. The study conducted by Michaud et al. ${ }^{18}$ compared the irradiance and the distribution of the wavelengths of LCUs of different generations, concluding that poly-wave LEDs have an inhomogeneous irradiance profile. Furthermore, Cardoso et al. ${ }^{8}$ suggested that the wavelength emitted by the single-wave LEDs covers a narrow part of the LucirinTPO absorption wavelength; it is possible that the use of a high radiant emittance for a longer period of time may have led to the delivery of higher radiance exposure values within the narrow wavelength range capable of exciting Lucirin-TPO. In this study, based on the time and power for each apparatus, the irradiance values were concluded as $48 \mathrm{~J} / \mathrm{cm}^{2}$ for the single-wave LUC and $40 \mathrm{~J} / \mathrm{cm}^{2}$ for the poly-wave LCU. These facts may be the reasons for no differences between the LEDs used in this study. In other study, differences in color change were found between single-wave and poly-wave LUCs, likely because the composite resins had been manipulated in the laboratory and the LEDs were used with a $20 \mathrm{~J} / \mathrm{cm}^{2}$ radiant exposure. ${ }^{2}$ In addition, it is suggested that the type of yellowness of a photoinitiator may influence the results when different LCUs are compared. ${ }^{2}$

When comparing the studied composite resins, the two composites with camphorquinone and lucirin-TPO (Tetric N-Ceram and Vit-l-escence) had less overall color change $(\Delta \mathrm{E})$ when compared to the composite resin that only had camphorquinone (FiltekZ350XT). Farah and Elwi ${ }^{19}$ also found that Tetric N-Ceram resin demonstrated significantly less color change when compared to FiltekZ350XT. The nanohybrid composite demonstrated greater color stability after AAA when compared to the nano-filled composite, ${ }^{3,20}$ indicating that the combination of camphorquinone and TPO reduced yellowness and color change when compared to the camphorquinone only system. This was expected because the yellowness appearance of TPO increased the TPO radiant exposure absorbtion within the first $1 \mathrm{~mm}$ thickness, which also reduced the light being transmitted to the bottom, at least up to a thickness of $2 \mathrm{~mm} .^{3}$ Furthermore, the effect of the addition of TPO was concentration-dependent, ${ }^{3}$ explaining the differences of overall color change between Vit-1escence and Tetric N-Ceram, since manufacturers did not cite the concentration of lucirin-TPO as cited above. FiltekZ350XT presents only camphorquinone as a photoinitiator; additionally, it contains TEGDMA, which has a greater capacity for water absorption relative to other monomers, such as Bis-GMA and UDMA making the resin more susceptible to overall color change, ${ }^{21}$ explaining the results found in this present study. Another study demonstrated that TPO-containing resins exhibited better color stability when compared to the camphorquinone-containing resins when pigmented in black tea and distilled water. ${ }^{22}$ The camphorquinone + TPO association also showed better color stability when 
compared to camphorquinone alone, after 30 days of storage in water. ${ }^{23}$

Regarding other color parameters, $\Delta \mathrm{L}$ and $\Delta \mathrm{a}$ had more stability than $\Delta \mathrm{b}$, since the challenge used may have a greater affecton coordinate $b$, such as occurred with another study. ${ }^{20}$ Furthermore, when comparing the resin composites, there were no differences in $\Delta \mathrm{L}$ and similar differences found in $\Delta \mathrm{a}$, regardless of the LUC used. The AAA caused an increase in the value of $\Delta \mathrm{b}$, making specimens more saturated/ chromatic (positive $\Delta b$ values) tending to yellow. It is thought that UV radiation is more related to a physical modification of the material surface, including loss of cohesion between fillers, pigments, and the polymeric material. ${ }^{5}$ The difference in color was visually noticed. However, other studies found that, after photodegradation by UV aging, photoinitiators other than CQ had a photo-bleaching effect of $>$ L (bleach) and $<$ b (less yellow), which could be justified by the absorption of water into the polymer during aging, increasing light scattering. ${ }^{2}$

The literature has shown studies where composites were manipulated in the laboratory to quantify photoinitiators and standardize the composite resin samples. ${ }^{2,3,8,10,22,23}$ However, this does not match what the dentist experiences every day in the office, demonstrating the high clinical relevance of the present study by using resins commonly found in the dental market.

A limitation of the present study was that the irradiance used by the LCUs was not the same. The two opaque composite resins used in this study were photoactivated for 40 seconds as suggested by the manufacturers'. The same polymerization time was used by Salgado et al. ${ }^{23}$ when using a single-wave LED and for Albuquerque et al. ${ }^{24}$ using a poly-wave LED. It is known that composite resins when photoactivated by an LCU, must receive sufficient energy and within the appropriate wavelength range so that an effective photopolymerization is produced..$^{18}$ Furthermore, Illie and Hickel $^{6}$ found that the minimum irradiation necessary for a $2 \mathrm{~mm}$ increment was reached only for polymerization times of 20 seconds and 40 seconds using a single-wave LCU. Moreover, it is still necessary that more studies are conducted to evaluate other properties of composite resins, such as roughness and brightness when using different generations of LEDs.

\section{CONCLUSION}

In general, the LED with multiple wavelengths influenced the microhardness of only one composite resin containing lucerin-TPO photoinitiator after AAA. The overall color change seems to be more influenced by the composition of composite resins and less influenced by the type of LED used. Although Filtek Z350XT presented high values of microhardness, it also presented a great color change after AAA. The polywaveLED LCU provided an ideal polymerization of commercial composite resins with Lucirin TPO when submitted to an aggressive challenge, whereas the single-wave LED LUC may be used since an adequate irradiance was applied. The knowledge of the behavior of composite resins with regards to the polymerization mechanism could prevent restorations from becoming more susceptible to color change and have a reduction of mechanical strength.

\section{CLINICAL SIGNIFICANCE}

The knowledge of composite resin with deficiencies in the polymerization mechanism could contribute to prevent restorations to become more susceptible to color change and reduction of the mechanical strength.

\section{REFERENCES}

1. Porto IC, Soares LE, Martin AA, Cavalli V, Liporoni PC. Influence of the photoinitiator system and light photoactivation units on the degree of conversion of dental composites. Braz Oral Res 2010 Oct-Dec;24(4):475-481.

2. Oliveira DC, Rocha MG, Gatti A, Correr AB, FerracaneJL, Sinhoret MA. Effect of different photoinitiators and reducing agents on cure efficiency and color stability of resin-based composites using different LED wavelengths. J Dent 2015 Dec;43(12):1565-1572.

3. Oliveira DC, Rocha MG, Correa IC, Correr AB, Ferracane JL, Sinhoreti MA. The effect of combining photoinitiator systems on the color and curing profile of resin-based composites. Dent Mater 2016 Oct;32(10):1209-1217.

4. Price RB, Labrie D, Rueggeberg FA, Sullivan B, Kostylev I, Fahey J. Correlation between the beam profile from a curing light and the microhardness of four resins. Dent Mater 2014 Dec;30(12):1345-1357.

5. Catelan A, Suzuki TYU, Becker F JR, Briso ALF, dos Santos $\mathrm{PH}$. Influence of surface sealing on color stability and roughness of composite submitted to ultravioet-accelerated aging. J Investig Clin Dent 2017 May;8(2).

6. Ilie N, Hickel R. Can CQ be completely replaced by alternative initiators in dental adhesives? Dent Mater J 2008 Mar;27(2):221-228.

7. Santini A, Miletic V, Swift MD, Bradley M. Degree of conversion and microhardness of TPO-containing resin-based composites cured bypolywaveandmonowaveLED units. J Dent 2012 Jul; 40(7):577-584.

8. Cardoso KA, Zarpellon DC, Madruga CF, Rodrigues JA, Arrais CA. Effects of radiant exposure values using second and third generation light curing units on the degree of conversion of a lucirin-based resin composite. J Appl Oral Sci 2017 Mar-Apr;25(2):140-146.

9. Ferracane JL. Resin-based composite performance: are there some things we can't predict? Dent Mater 2013 Jan;29(1):51-58. 
10. Schneider LF, Cavalcante LM, PrahlSA, Pfeifer CS, Ferracane JL. Curing efficiency of dental resin composites formulated with camphorquinone or trimethylbenzoyl-diphenyl-phosphine oxide. Dent Mater 2012 Apr;28(4):392-397.

11. Sim JS, Seol HJ, Park JK, Garcia-Godoy F, Kim HI, Kwon YH. Interaction of LED light with coinitiator-containing composite resins: Effect of dual peaks. J Dent 2012 Oct;40(10):836-842.

12. Ferracane JL. Resin composite-State of the art. Dent Mater 2011 Jan;27(1):29-38.

13. Rosa RS, Balbinot CE, Blando E, Mota EG, Oshima HM, Hirakata L, et al. Evaluation of mechanical properties on three nanofilled composites. Stomatologija 2012;14(4):126-130.

14. Catelan A, Briso AL, Sundfeld RH, dos Santos PH. Effect of artificial aging on the roughness and microhardness of sealed composites. J Esthet Restor Dent 2010 Oct;22(5):324-330.

15. Reis AC, Castro DT, Schiavon MA, Silva LJ, Agnelli JAM. Microstructure and mechanical properties of composite resins subjected to accelerated artificial aging. Braz Dent J 2013 Nov-Dec;24(6):599-604.

16. Borges AB, Caneppele TMF, Luz M, Pucci CR, Torres CRG. Color stability of resin used for caries infiltration after exposure to different staining solutions. Oper Dent 2014 Jul-Aug;39(4):433-440.

17. Inokoshi S, Burrow MF, Kataumi M, Yamada T, Takatsu T. Opacity and color changes of tooth-colored restorative materials. Oper Dent 1996 Mar-Apr;21(2):73-80.

18. Michaud PL, Price RB, Labrie D, Rueggeberg FA, Sullivan B. Localised irradiance distribution found in dental light curing units. J Dent 2014 Feb;42(2):129-139.
19. Farah RI, Elwi H. Spectrophotometric evaluation of color changes of bleach-shade resin-based composites after staining and bleaching. J Contem Dent Pract 2014 Sep 1;15(5): 587-594.

20. Oliveira DC, Souza-Júnior EJ, Prieto LT, Coppini EK, Maia RR, Paulillo LA. Color stability and polymerization behavior of direct esthetic restorations. J EsthetRestor Dent 2014 JulAug;26(4):288-295.

21. Rey N, Benbachir N, Bortolotto T, Krejci I. Evaluation of the staining potential of a caries infiltrant in comparison to other products. Dent Mater J 2014;33(1):86-91.

22. Manojlovic D, Dramićanin MD, Lezaja M, Pongprueksa $\mathrm{P}$, Van Meerbeek B, Miletic V. Effect of resin and photoinitiator on color, translucency and color stability of conventional and low-shrinkage model composites. Dent Mater 2016 Feb;32(2):183-191.

23. Salgado VE, Borba MM, Cavalcante LM, Moraes RR, Schneider LF. Effect of photoinitiator combinations on hardness, depth of cure, and color of model resincomposites. J EsthetRestor Dent 2015 Mar-Apr;27(1):S41-48.

24. Albuquerque PP, Bertolo ML, Cavalcante LM, Pfeifer C, Schineider LF. Degree of conversion, depth of cure, and color stability of experimental dental composite formulated with camphorquinone and phenanthrenequinonephotoinitiators. J EsthetRestor Dent 2015 Mar-Apr;27(1):S49-57. 